\title{
Unidirectional Kondo scattering in layered $\mathrm{NbS}_{2}$
}

Edoardo Martino $\mathbb{D}^{1 凶}$, Carsten Putzke $\mathbb{D}^{2}{ }^{2}$, Markus König ${ }^{3}$, Philip J. W. Moll $\mathbb{D}^{2}$, Helmuth Berger ${ }^{1}$, David LeBoeuf ${ }^{4}$, Maxime Leroux $\mathbb{D}^{4}$, Cyril Proust $^{4}$, Ana Akrap id ${ }^{5}$, Holm Kirmse ${ }^{6}$, Christoph Koch ${ }^{6}$, ShengNan Zhang ${ }^{1,7}$, QuanSheng Wu ${ }^{1,7}$, Oleg V. Yazyev (iD ${ }^{1,7}$, László Forró ${ }^{1,8 凶}$ and Konstantin Semeniuk (D) $^{1 凶}$

Crystalline defects can modify quantum interactions in solids, causing unintuitive, even favourable, properties such as quantum Hall effect or superconducting vortex pinning. Here we present another example of this notion-an unexpected unidirectional Kondo scattering in single crystals of $2 \mathrm{H}-\mathrm{NbS}_{2}$. This manifests as a pronounced low-temperature enhancement in the out-of-plane resistivity and thermopower below $40 \mathrm{~K}$, hidden for the in-plane charge transport. The anomaly can be suppressed by the $c$-axisoriented magnetic field, but is unaffected by field applied along the planes. The magnetic moments originate from layers of 1T$\mathrm{NbS}_{2}$, which inevitably form during the growth, undergoing a charge-density-wave reconstruction with each superlattice cell (David-star-shaped cluster of $\mathrm{Nb}$ atoms) hosting a localised spin. Our results demonstrate the unique and highly anisotropic response of a spontaneously formed Kondo-lattice heterostructure, intercalated in a layered conductor.

npj 2D Materials and Applications (2021)5:86; https://doi.org/10.1038/s41699-021-00265-6

\section{INTRODUCTION}

Layered van der Waals materials, such as transition metal dichalcogenides (TMDs), have attracted major interest, thanks to their rich variety of ground states and the possibility of their exfoliation down to an atomically thin level, which remarkably modifies their electronic properties ${ }^{1,2}$. Recent observations of intriguing physics in artificially assembled heterostructures highlight the importance of interlayer interactions. Examples include the outstanding stability of interlayer excitons in semiconducting $\mathrm{TMDs}^{3}$, and strongly correlated states in twisted bilayer systems ${ }^{4}$. Relevant aspects of the inter-plane coupling can be deduced by probing out-of-plane charge transport, even in bulk materials ${ }^{5}$. However, enforcing the current flow strictly along the $c$ axis can be rather challenging due to the crystals' common flake-like appearance and their propensity for delamination. Such a pitfall can distort the measurement results by orders of magnitude, as demonstrated in our recent study of microstructured samples of $1 \mathrm{~T}-\mathrm{TaS}_{2}$ with a well-defined current flow $^{6}$. This observation motivates a careful re-examination of the out-of-plane charge transport properties in this class of materials by adopting the latest state-of-the-art for quantum matter microfabrication ${ }^{7}$.

Here we present data on the out-of-plane electrical resistivity of bulk monocrystalline $2 \mathrm{H}-\mathrm{NbS}_{2}$. This material is one of the three known structural variants of layered $\mathrm{NbS}_{2}$. The two other polytypes are $3 R$ and $1 T$, the latter occurring only in atomically thin form ${ }^{8,9}$. As illustrated in Fig. $1 \mathrm{a}, 1 \mathrm{~T}-\mathrm{NbS}_{2}$ consists of cornersharing octahedral $\mathrm{NbS}_{6}$ cells. Layers of $2 \mathrm{H}-$ and $3 \mathrm{R}-\mathrm{NbS}_{2}$ (Fig. 1b, c) both contain $\mathrm{NbS}_{6}$ units of trigonal prismatic geometry, but exhibit different stacking configurations. The $1 \mathrm{~T}$ polytype has been attracting interest recently as a candidate for realising a twodimensional magnetic system ${ }^{10,11} \cdot 2 \mathrm{H}-\mathrm{NbS}_{2}$ has been actively featured in the literature due to a superconductivity below $6 \mathrm{~K}$, proposed to have a multiband character. It also does not show any charge-density-wave (CDW) order, which is uncommon for metallic TMDs ${ }^{12-17}$. Another distinguishing feature of $2 \mathrm{H}-\mathrm{NbS}_{2}$ is its non-trivial synthesis procedure. This polytype is thermodynamically stable in a relatively narrow range of temperatures and reactant stoichiometries ${ }^{18-20}$. Crystals formed during hightemperature growth must be rapidly quenched in order to capture $2 \mathrm{H}-\mathrm{NbS}_{2}$ in a metastable room-temperature state. However, X-ray diffraction studies have shown that the resultant material has up to $18 \%$ of pairs of neighbouring layers stacked in a 3R-like manner ${ }^{21,22}$. Additionally, diffuse X-ray scattering experiments ${ }^{22}$ revealed weak traces of the $\sqrt{13} \times \sqrt{13}$ CDW reconstruction, which appears as a triangular superlattice of David-starshaped clusters defined by $13 \mathrm{Nb}$ atoms ${ }^{23}$. Such a reconstruction is not expected for pure $2 \mathrm{H}-\mathrm{NbS}_{2}$ or $3 \mathrm{R}-\mathrm{NbS}_{2}$. Earlier theoretical investigations have predicted $1 \mathrm{~T}-\mathrm{NbS}_{2}$ to be particularly prone to developing such a CDW order $^{10,11}$. One can therefore conclude that single crystals of $2 \mathrm{H}-\mathrm{NbS}_{2}$ contain rare, atomically thin inclusions of the $1 \mathrm{~T}$ polytype.

Our study of $2 \mathrm{H}-\mathrm{NbS}_{2}$ revealed a remarkably strong lowtemperature anomaly in the compound's out-of-plane resistivity $\left(\rho_{c}\right)$, manifesting as a minimum at around $40 \mathrm{~K}$, followed by a pronounced upturn upon further cooling. The feature is simultaneously invisible in the in-plane resistivity $\left(\rho_{a b}\right)$, and shows a highly anisotropic response to magnetic field. Neither $2 \mathrm{H}-\mathrm{NbSe}_{2}$ nor $3 \mathrm{R}-\mathrm{NbS}_{2}$ display such an anomaly, implying that the phenomenon is linked to the structural defects specific to $2 \mathrm{H}$ $\mathrm{NbS}_{2}$. 1T-NbS 2 , layers of which are one of such defects, were predicted to form a lattice of unpaired localised spins located at the centre of each David-star CDW superlattice cluster ${ }^{10,11}$. We argue that planes of magnetic moments, hosted by the inclusions of $1 \mathrm{~T}-\mathrm{NbS}_{2}$, cause a Kondo effect observable only when the current flows across these planes.

\footnotetext{
${ }^{1}$ Institute of Physics, École Polytechnique Fédérale de Lausanne (EPFL), CH-1015 Lausanne, Switzerland. ${ }^{2}$ Institute of Materials Science and Engineering, École Polytechnique Fédérale de Lausanne (EPFL), CH-1015 Lausanne, Switzerland. ${ }^{3}$ Max Planck Institute for Chemical Physics of Solids, 01187 Dresden, Germany. ${ }^{4}$ Laboratoire National des Champs Magnétiques Intenses (LNCMI-EMFL), CNRS, UGA, UPS, INSA, Grenoble/Toulouse, France. ${ }^{5}$ Department of Physics, University of Fribourg, CH-1700 Fribourg, Switzerland. ${ }^{6}$ Department of Physics, Humboldt University of Berlin, Berlin 12489, Germany. ${ }^{7}$ National Center for Computational Design and Discovery of Novel Materials MARVEL, École Polytechnique Fédérale de Lausanne (EPFL), CH-1015 Lausanne, Switzerland. ${ }^{8}$ Stavropoulos Center for Complex Quantum Matter, University of Notre Dame, Notre Dame 46556

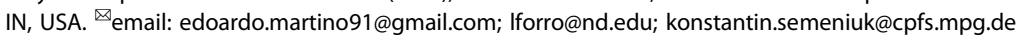


(a)

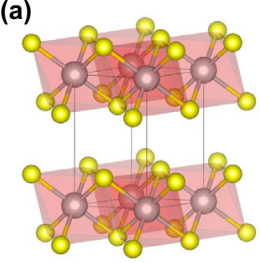

(b)

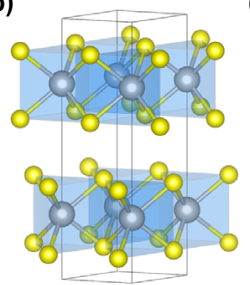

$1 \mathrm{~T}-\mathrm{NbS}_{2}$ (c)

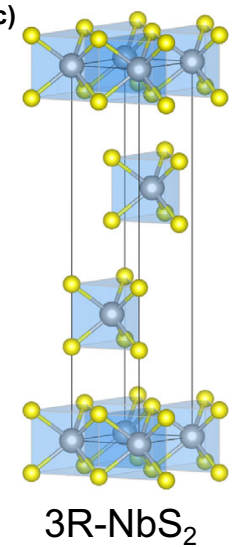

Fig. 1 Polytypes of $\mathrm{NbS}_{2}$. Crystalline lattices of $1 \mathrm{~T}-\mathrm{NbS}_{2}(\mathbf{a}), 2 \mathrm{H}-$ $\mathrm{NbS}_{2}$ (b) and 3R-NbS 2 (c). The corresponding 1-, 2- and 3-layer unit cells are marked with black wireframes. The compass shows the directions of the principal axes for all three structures. $2 \mathrm{H}-\mathrm{NbS}_{2}$ and $3 \mathrm{R}-\mathrm{NbS}_{2}$ share the same structure in the $a-b$ plane, but have different stacking of layers along the $c$ axis. For $3 \mathrm{R}-\mathrm{NbS}_{2}$, only one $\mathrm{Nb}$ atom with 6 nearest $\mathrm{S}$ atoms are shown for the middle two layers, for a clearer illustration of the stacking. Images produced with VESTA $^{57}$.

\section{RESULTS}

\section{Resistivity anisotropy}

Optimisation of sample geometry with focused ion beam (FIB) micro-milling greatly improves charge transport study precision ${ }^{6,7}$. Using this approach, we shaped single crystals into samples with well-defined, few micron-thick and -wide current channels, oriented along the two principal directions: normal and parallel to the atomic planes (Fig. 2a shows a sample of $2 \mathrm{H}-\mathrm{NbS}_{2}$ produced this way). Such a design allowed simultaneous measurement of both $\rho_{a b}$ and $\rho_{c}$ via the four-point technique. Probing $\rho_{c}$ on two segments of different surface-to-volume resulted in mutually consistent values, allowing us to ensure that our results were not distorted by the presence of surface-related effects or macroscopic defects.

Figure $2 \mathrm{~b}$ shows the plots of $\rho_{a b}$ and $\rho_{c}$ of $2 \mathrm{H}-\mathrm{NbS}_{2}$ against temperature $(T)$, as well as their ratio in the inset. Note that in contrast to the earlier study which reports an anisotropy of the order of 1000 (ref. ${ }^{24}$ ), our measured value was as low as 10 at room temperature, monotonically increasing to 180 on cooling. As it was shown for the case of $1 \mathrm{~T}-\mathrm{TaS}_{2}$ using finite element simulations ${ }^{6}$, such an overestimate by the previous study could be a result of a nonoptimised measurement geometry and an incorrect prior assumption that the anisotropy is very large. While $\rho_{a b}$ has a conventional metallic temperature dependence, $\rho_{c}$ is also metallic, but shows a few noteworthy features. First, the residual out-of-plane resistivity is very high, presumably due to a significant concentration of static defects. Second, $\rho_{c}$ approaches saturation in the high-temperature region. This flattening of resistivity may be attributed to the mean free paths decreasing to the point of becoming comparable to the interlayer separation, a concept known as the Mott-loffe-Regel limit ${ }^{25}$. Third, at low temperatures, $\rho_{c}$ displays a minimum at around $40 \mathrm{~K}$, with a major upturn at lower temperatures. No corresponding feature exists in $\rho_{a b}$ (in agreement with the previous results ${ }^{26}$ ). All studied samples of $2 \mathrm{H}-\mathrm{NbS}_{2}$ showed qualitatively identical behaviour, with slight differences in the absolute values of resistivityrelated to slight impurity content variations-and temperatures of the minimum distributed in the $30-40 \mathrm{~K}$ range.

We compared $\rho_{c}$ of $2 \mathrm{H}-\mathrm{NbS}_{2}$ to that of the isostructural and isovalent compound $2 \mathrm{H}-\mathrm{NbSe}_{2}$ (dashed line in Fig. 2b). The latter material did not exhibit a similar low-temperature anomaly. Based on the nominal lattice parameters, density functional theory calculations predict that the two compounds will have nearly
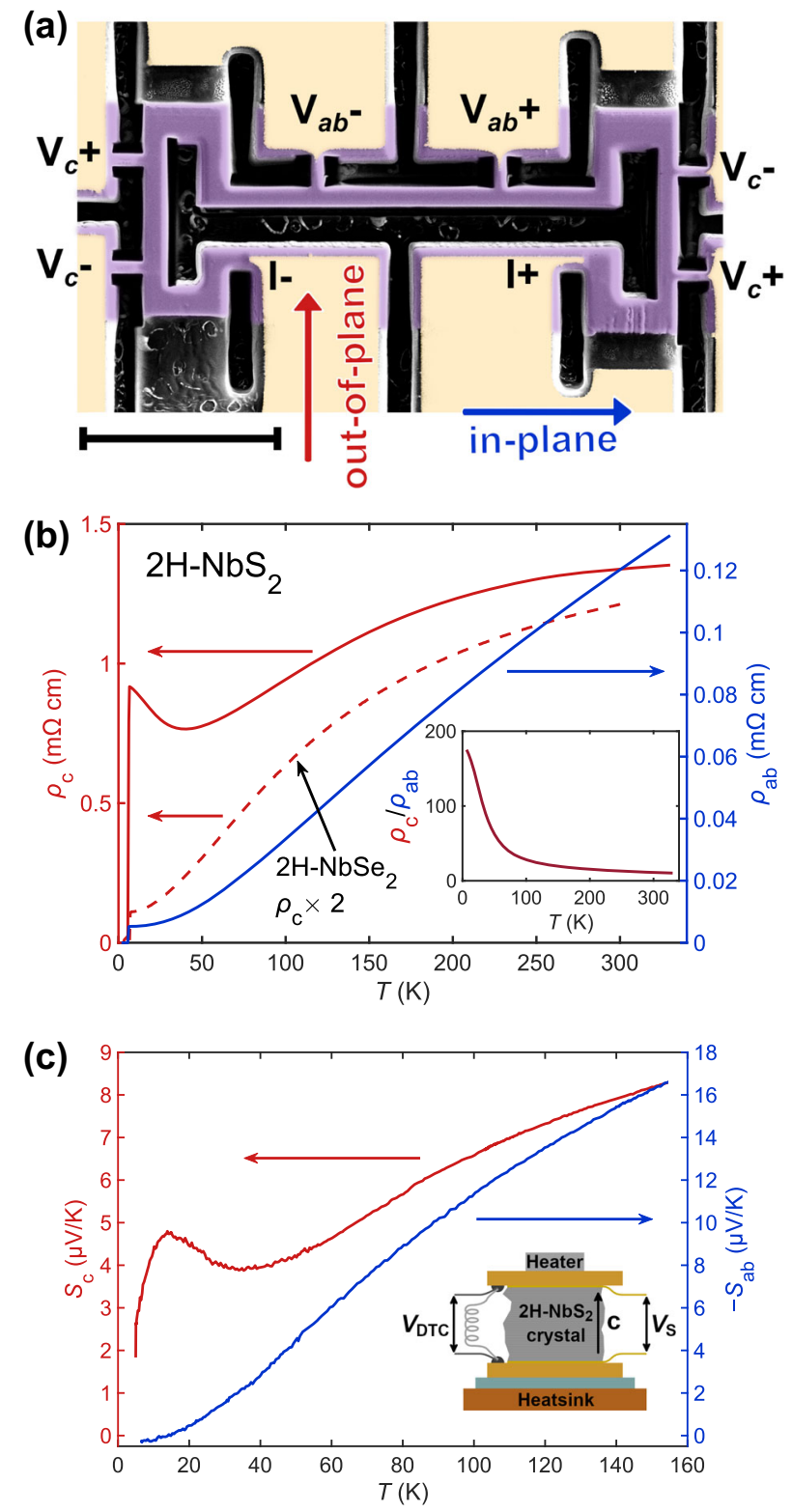

Fig. 2 Interlayer charge dynamics in $\mathbf{2} \mathbf{H}-\mathbf{N b S}_{2}$. a Scanning electron microscope image of a $2 \mathrm{H}-\mathrm{NbS}_{2}$ sample, structured with focused ion beam for accurate resistivity anisotropy measurements. False colouring is used: purple - crystal, beige - gold film. The scale bar in the bottom left is $20 \mu \mathrm{m}$ long. The current sourcing (I) and voltage probing (V) electrodes are labelled. b Plots of the in-plane $\left(\rho_{a b}\right.$, blue) and out-ofplane $\left(\rho_{c}\right.$ red) resistivities of $2 \mathrm{H}-\mathrm{NbS}_{2}$ against temperature $(T)$. The dashed red line stands for the out-of-plane resistivity of $2 \mathrm{H}-\mathrm{NbSe}_{2}$, scaled by a factor of 2 . Resistivity anisotropy of $2 \mathrm{H}-\mathrm{NbS}_{2}$ is plotted in the inset. c Seebeck coefficients of $2 \mathrm{H}-\mathrm{NbS}_{2}$ for the out-of-plane $\left(S_{c}\right)$ and in-plane $\left(S_{a b}\right)$ directions as functions of temperature, measured on bulk single crystals (note that $S_{a b}$ is negative). The setup for measuring $S_{c}$ is illustrated schematically. The crystal was approximately $1 \mathrm{~mm}$ long in the $c$ axis direction (indicated in the drawing), and $2-3 \mathrm{~mm}$ long laterally. The value of $S_{c}$ is the ratio of the voltage across the sample $\left(V_{s}\right)$ and the thermal gradient across it, determined from the differential thermocouple voltage $\left(V_{\text {DTC }}\right)$. The sample sat between two copper plates, which homogenised temperature at its two faces and was electrically decoupled from the heatsink by a thin sapphire plate.

identical electronic band structures (see Supplementary Note 1 and Supplementary Figs. 1 and 2). We therefore conclude that the upturn of $\rho_{c}$ of $2 \mathrm{H}-\mathrm{NbS}_{2}$ is not intrinsic to the nominal structure of the compound, but is caused by crystalline lattice defects. 


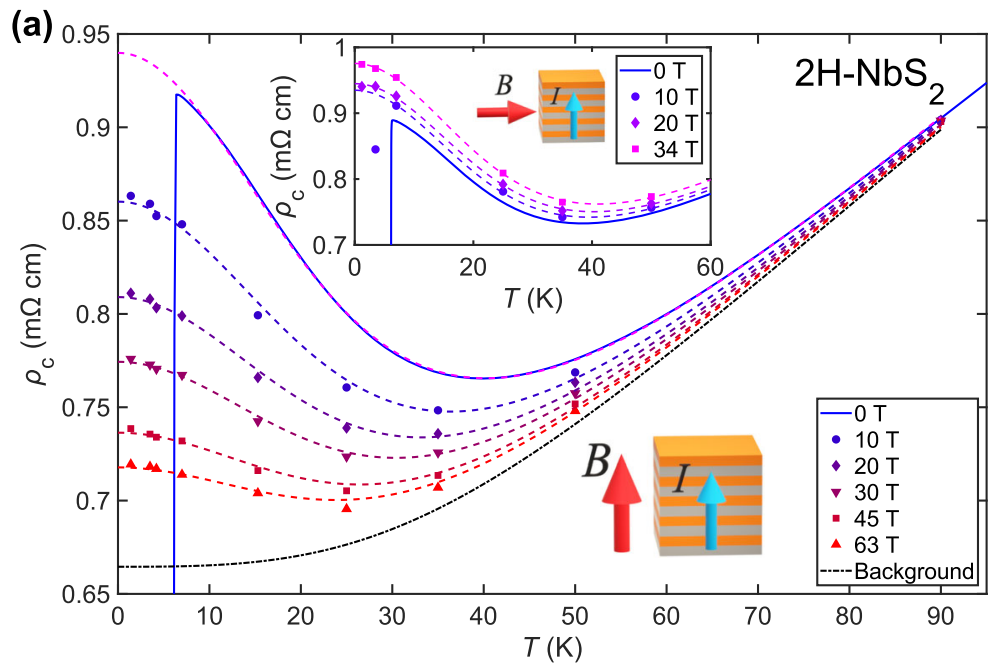

(b)

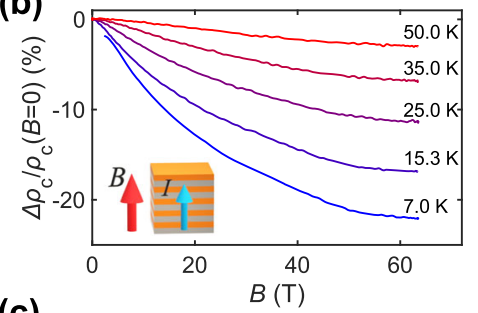

(c)

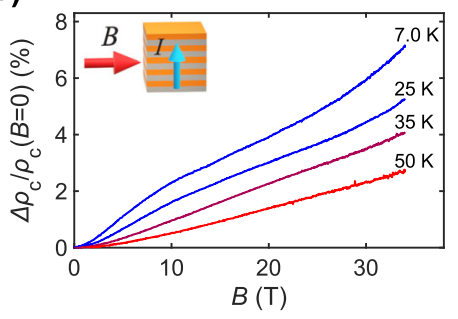

Fig. 3 Out-of-plane magnetotransport in $\mathbf{2} \mathbf{H}-\mathbf{N b S}_{\mathbf{2}}$. a Out-of-plane resistivity $\left(\rho_{c}\right)$ as a function of temperature $(T)$ for various longitudinal (main plot) and transverse (inset) magnetic fields $(B)$. Solid lines and markers represent the measured data. Dashed lines in the main plot are fits according to the numerical renormalisation group theory of Kondo effect. The fitted model includes a field-independent contribution due to the residual temperature-independent resistance as well as the electron-phonon scattering, described by the Bloch-Grüneisen formula (dash-dot line). Dashed lines in the inset are guides for the eye. The two datasets were collected using different samples, which explains the slight resistivity mismatch for zero field. b, c Relative changes in $\rho_{c}$, under out-of-plane (b) and in-plane (c) magnetic fields.

\section{Seebeck coefficient anisotropy}

The Seebeck coefficient $(S)$ is a useful quantity for sensitively probing energy landscape variations near Fermi level. The opencircuit voltage generated by the thermal gradient is unaffected by the presence of static defects such as vacancies or non-magnetic stacking faults. On the other hand, the Seebeck coefficient is a function of the energy dependence of the conduction electron scattering rate. This is then strongly affected by the occurrence of resonance peaks in the density of states close to the chemical potential. Seebeck coefficient of $2 \mathrm{H}-\mathrm{NbS}_{2}$ revealed a prominent peak at approximately $15 \mathrm{~K}$, appearing only for the out-of-plane thermal gradient (Fig. 2c). The phonon drag phenomenon produces a similar feature in the temperature dependence. However, it manifests only in conductors with long phonon and charge carrier mean free paths-such as semimetals or extremely pure metalswhere momentum-conserving scattering is dominant ${ }^{27}$. This is highly unlikely for $2 \mathrm{H}-\mathrm{NbS}_{2}$, as its high content of static defects should clearly favour momentum-relaxing scattering. Furthermore, absence of the corresponding $S_{a b}$ peak rules out the phonon drag from the possible $S_{c}$ anomaly origins. An alternative interpretation of the peak, the Kondo effect, will be discussed further below.

\section{High-field magnetotransport}

The out-of-plane resistivity anomaly of $2 \mathrm{H}-\mathrm{NbS}_{2}$ demonstrated a particularly curious response to magnetic fields. As can be seen in Fig. 3a, the transverse and longitudinal out-of-plane magnetoresistances of the material are strikingly different. Applying the field along the $c$ axis suppresses the resistivity upturn, shifting the minimum to lower temperature. Yet even at $63 \mathrm{~T}$ the anomaly is still present. Consequently, at $50 \mathrm{~K}$ and below, $\rho_{c}$ decreases when magnetic field is increased, with signs of saturation appearing around $50 \mathrm{~T}$ (Fig. $3 \mathrm{~b}$ ). In contrast, in transverse magnetic field, $\rho_{c}$ behaves as a more typical orbital magnetoresistance, common to metals. It is positive, and about three times weaker in magnitude than the longitudinal one (Fig. 3c) without significantly affecting the shape of the upturn in $\rho_{c}(T)$.

In order to emphasise the observed phenomenon's highly anisotropic nature, we also report the in-plane magnetotransport of $2 \mathrm{H}-\mathrm{NbS}_{2}$ up to $14 \mathrm{~T}$. The magnetoresistance is weak for all field directions, as can be seen in Fig. 4 a, but similarly to $\rho_{c}, \rho_{a b}$ is also reduced by the field along the $c$ axis (clearly depicted in Fig. 4b), which could be a trace of the same anomaly. The in-plane fielddependence of $\rho_{a b}$ (Fig. 4c) is likely governed by orbital effects, like in the case of $\rho_{c}$.

\section{DISCUSSION}

The question of the origin of the anomalies of $\rho_{c}$ in $2 \mathrm{H}-\mathrm{NbS}_{2}$ will now be addressed. A number of phenomena could result in a finitetemperature resistivity minimum in a metal. Resistivity upturns can be caused by electron-electron interactions in the presence of static disorder ${ }^{28-30}$. However, the corresponding quantum mechanical correction is either weakly enhanced by a magnetic field, or is effectively field-independent. A closely linked phenomenon of weak localisation (WL) is also known to produce an additional contribution to resistivity at low temperatures ${ }^{31}$. In this scenario, when a series of scattering events cause an electron to follow a closed path, quantum interference favours the net backward scattering over the forward one. Magnetic flux threading these scattering loops shifts the phases of the wave functions, diminishing the effect. In our case, when electrons are scattered between different planes, the closed paths should have comparable projections along the in-plane and out-of-plane directions. However, the upturn is only influenced by the c-axis-oriented field, contradicting the WL-based interpretation. A metal-insulator transition or conduction based on a thermally activated hopping between defects ${ }^{32}$ would cause a divergence of $\rho_{c}$ at the lowest temperatures, which was not the case. The possibility of quantum tunnelling playing a significant role is ruled out based on a linear relation between current and voltage (Supplementary Note 2 and Supplementary Fig. 3). Resistivity upturns have also been observed in strongly doped cuprate superconductors ${ }^{33,34}$. In those materials, the effect is believed to be caused by scattering from magnetic droplets forming around non-magnetic impurities. This interpretation, however, relies on the existence of strong electronic correlations, and therefore does not apply to our system.

Finite-temperature resistivity minimum in a metal is also a wellknown signature of the Kondo effect, a scattering of conduction electrons of dilute localised magnetic moments ${ }^{35}$. Besides the upturn, the characteristic features of the phenomenon, observable in charge transport, include a negative curvature of $\rho_{c}(T)$ at the 

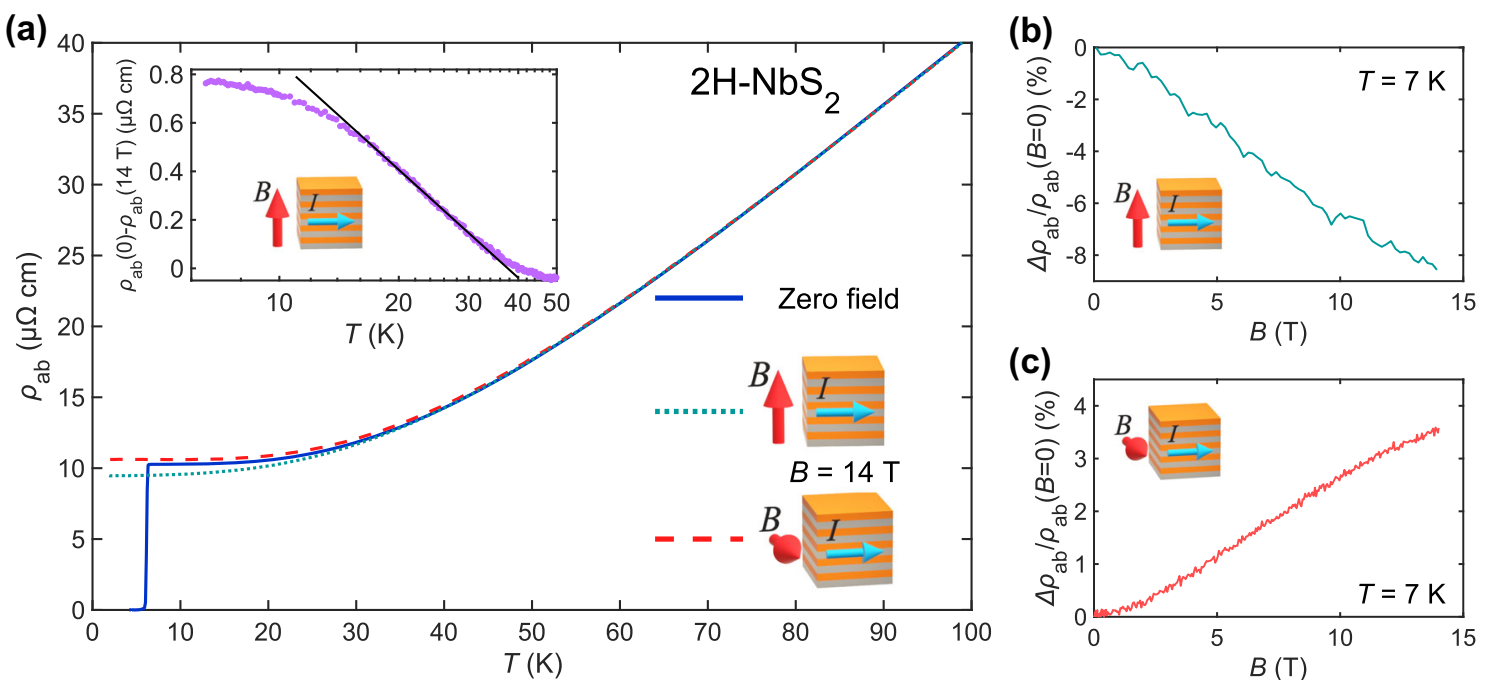

(c)

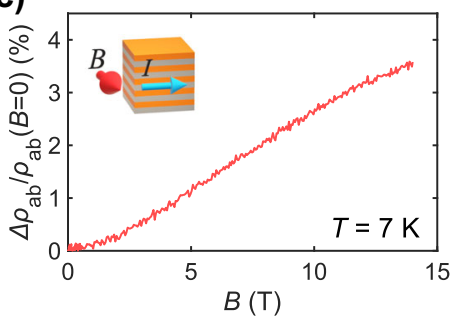

Fig. 4 In-plane magnetotransport in $\mathbf{2} \mathbf{H}-\mathbf{N b S}_{\mathbf{2}}$. a In-plane resistivity $\left(\rho_{a b}\right)$ as a function of temperature $(T)$ at zero and $14 \mathrm{~T}$ magnetic field $(B)$. The change of $\rho_{a b}$ between zero field and 14 T (out-of-plane field) is plotted in the inset on a logarithmic $T$ scale. The black line in the inset corresponds to the logarithmic temperature dependence. b, c In-plane magnetoresistance for the out-of-plane (b) and in-plane (c) field.

lowest temperatures and a suppression of the upturn by magnetic field, which causes spin-flip scattering to become inelastic ${ }^{36,37}$. The observed peak in the Seebeck coefficient is also characteristic to dilute and concentrated spin systems, including Kondo lattices ${ }^{38,39}$. It originates from the resonant scattering in the Kondo channel at the Fermi level. Above the Kondo temperature, $T_{\mathrm{K}}$, the resonance is smeared out, and depending on the specifics of a system, the peak in $S$ appears at a temperature between 0.3 and $0.9 T_{\mathrm{K}}$ (refs. ${ }^{39,40}$ ). When temperature is low enough, the localised spins are screened and the excitations obey simple power laws, like those of a Fermi liquid. For example, $S$ varies as $T /$ $T_{\mathrm{K}}$ for $T / T_{\mathrm{K}}<0.1-0.15$ for several typical Kondo alloys in the dilute, single impurity limit ${ }^{41}$.

Measurements of $\rho_{c}$ under high pressure, presented in Supplementary Note 3 and Supplementary Fig. 4, show that the upturn remains extremely robust up to the highest achieved pressure of $1.9 \mathrm{GPa}$. Applying the pressure weakly shifts the minimum of $\rho_{c}$ up in temperature. This is consistent with the behaviour expected from Kondo systems ${ }^{42,43}$.

We therefore argue that scattering of magnetic impurities is the most fitting explanation of our observations. The temperature dependence of $\rho_{c}$ in $2 \mathrm{H}-\mathrm{NbS}_{2}$ is consistent with the one expected from the numerical renormalisation group (NRG) theory calculations for Kondo effect ${ }^{36,44}$, as illustrated by the fit in Fig. 3a. We modelled $\rho_{c}$ with a sum of three contributions: a temperatureindependent residual resistivity $\rho_{0}$, an electron-phonon scattering term $\rho_{\mathrm{e}-\mathrm{p}}$ (captured by the Bloch-Grüneisen formula) and the Kondo term $\rho_{\mathrm{K}}$ (the only magnetic-field-dependent term), for which we used the common empirical expression closely following the results of the NRG theory ${ }^{36,44}$ :

$\rho_{\mathrm{K}}(T)=\rho_{\mathrm{K} 0}\left(1+\left(2^{1 / a}-1\right)\left(T / T_{\mathrm{K}}\right)^{2}\right)^{-a}$.

The fitting procedure is described in more detail in Supplementary Note 4, with the help of Supplementary Figs. 5 and 6 and Supplementary Table 1.

This explanation immediately raises a question regarding the nature of our system's magnetic impurities. The standard scenario where magnetic atoms are uniformly distributed clearly does not fit our picture. Doping $2 \mathrm{H}-\mathrm{NbS}_{2}$ with Fe results in the upturn observable in $\rho_{a b}$ as well as the disappearance of superconductivity $^{45}$. Additionally, the undoped material does not display a corresponding signature in the heat capacity ${ }^{46}$. Lack of pronounced anomaly effects on $\rho_{a b}$ implies that the responsible defects take the form of sparse planes, extending along the layers. When the current then flows along the layers, only a small fraction of the conduction electrons move in close proximity to these planes. But for the out-of-plane current flow, effectively all charge carriers have to pass through them, resulting in a particularly strong influence. Although we observed planar irregularities in the crystalline lattice via transmission electron microscopy, their atomic structure could not be determined due to a limited resolution (see Supplementary Note 5 and Supplementary Figs. 7 and 8). Taking a closer look at the inplane magnetoresistance for the $c$-axis-oriented field reveals that the difference between $\rho_{a b}$ at 0 and $14 \mathrm{~T}$ increases linearly with respect to $\ln (T)$ between 30 and $15 \mathrm{~K}$ (Fig. 4a inset), which further supports our hypothesis. This means that a minute contribution of Kondo scattering is present in $\rho_{a b}$, but it is not strong enough to change the sign of the gradient of $\rho_{a b}(T)$.

The presence of $1 \mathrm{~T}-\mathrm{NbS}_{2}$ layers, evidenced by the characteristic CDW signatures ${ }^{22}$, offers a fascinating interpretation of our findings, illustrated in Fig. 5. As we mentioned in the introduction, the $\sqrt{13} \times \sqrt{13}$ CDW order, associated with the 1T polytype, forms a triangular superlattice of David-star-shaped clusters defined by $13 \mathrm{Nb}$ atoms ${ }^{23}$. The electronic structure of monolayer $1 \mathrm{~T}-\mathrm{NbS}_{2}$ as well as $1 \mathrm{~T}-\mathrm{NbSe}_{2}$ in such a configuration has been predicted to contain one very flat band around the Fermi level. This makes the materials susceptible to electronic instabilities like Mott localisation, with a concomitant magnetic order ${ }^{10,47,48}$. The referenced works found the ferromagnetic insulating state as the most stable, although others have proposed that such triangular lattices can host antiferromagnetic spin-liquid phases ${ }^{49,50}$. These magnetic planes play the role of scatterers in the Kondo effect, however, their concentration appears to be too low for a detection via magnetometry measurements. The described scenario is conceptually similar to the Kondo effect occurring in artificially fabricated magnetic tunnel junctions ${ }^{51,52}$, yet in our case the phenomenon is observed in a spontaneously formed system. The same kind of Kondo interaction has been very recently observed in scanning tunnelling spectroscopy studies of the $2 \mathrm{H} / 1 \mathrm{~T}$ or $1 \mathrm{H} / 1 \mathrm{~T}$ heterostructures of $\mathrm{NbSe}_{2}$ (ref. ${ }^{53}$ ), $\mathrm{TaS}_{2}$ (ref. ${ }^{54}$ ) and $\mathrm{TaSe}_{2}$ (ref. ${ }^{55}$ ), grown by molecular beam epitaxy. One outstanding question is the anomaly's markedly different response to the two orientations of magnetic field. This difference is probably coming from the localised electron's highly anisotropic $g$-factor, causing a very small spin splitting (less than $T_{\mathrm{K}}$ ), 


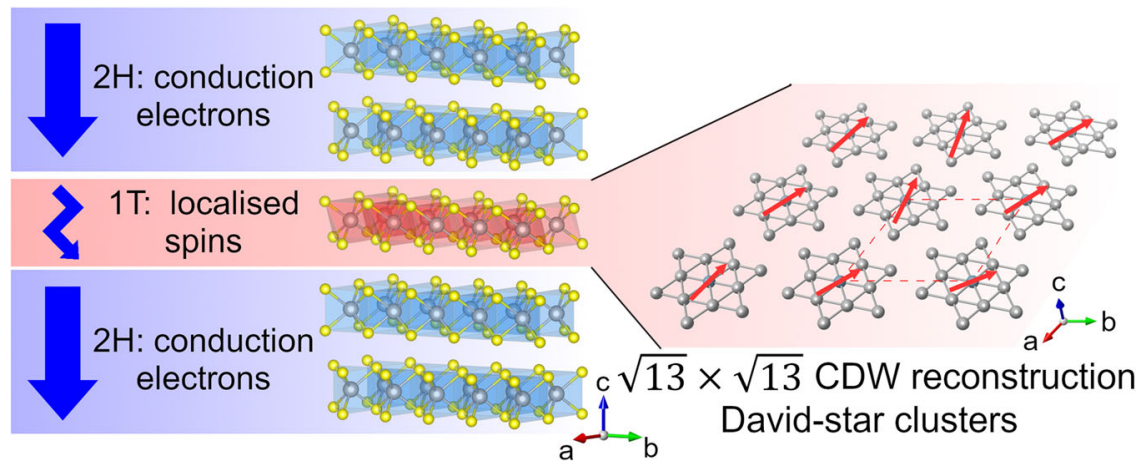

Fig. 5 1T-NbS 2 inclusions in $\mathbf{2} \mathbf{H}-\mathbf{N b S}_{2}$. Schematic visualisation of the proposed interpretation of the observed out-of-plane charge transport anomaly. The $2 \mathrm{H}-\mathrm{NbS}_{2}$ crystal (structure in the centre) contains inclusion layers of $1 \mathrm{~T}-\mathrm{NbS}_{2}$ (highlighted in red). $1 \mathrm{~T}-\mathrm{NbS} \mathrm{S}_{2}$ undergoes a $\sqrt{13} \times \sqrt{13}$ charge-density-wave (CDW) reconstruction. The $\mathrm{Nb}$ atoms in the CDW state are arranged into David-star-shaped clusters, superlattice of which is depicted on the right (only $\mathrm{Nb}$ atoms are shown, the dashed line marks the unit cell after the reconstruction). Each cluster contains an unpaired localised spin at the centre (red arrows on the right). The orientations of spins in the illustration are arbitrary and are not meant to suggest any particular ordering. This array of localised magnetic moments causes the itinerant electrons in the $2 \mathrm{H}-\mathrm{NbS} \mathrm{S}_{2}$ bulk to experience Kondo scattering during the out-of-plane current flow (blue arrows).

but could also be related to the magnetic ordering. Sizeable anisotropy of the $g$-factor is expected for systems with strong spin-orbit coupling, such as TMDs ${ }^{50}$.

Since $2 \mathrm{H}-\mathrm{NbS}_{2}$ is known to contain frequent 3R-like stacking faults, it is natural to ask whether the anomaly is somehow caused by the inclusions of $3 \mathrm{R}-\mathrm{NbS}_{2}$. We measured the latter compound's out-of-plane resistivity, and while the corresponding temperature dependence was surprisingly found to be nonmetallic, the extremely weak reaction of the interlayer conduction to the longitudinal magnetic field $\left(\Delta \rho_{c} / \rho_{c} \approx 0.1 \%\right.$ at $\left.14 \mathrm{~T}\right)$ was incompatible with the behaviour observed in $2 \mathrm{H}-\mathrm{NbS}_{2}$ (see Supplementary Note 6 as well as Supplementary Fig. 9 for the relevant data on $3 \mathrm{R}-\mathrm{NbS}_{2}$ ). The abundance of these stacking faults could explain the high residual component of $\rho_{c}$. The current understanding is that the poor conductivity of $3 \mathrm{R}-\mathrm{NbS}_{2}$ is not intrinsic, but rather originates from the disorder due to selfintercalated $\mathrm{Nb}$ atoms ${ }^{56}$. However, in $2 \mathrm{H}-\mathrm{NbS}_{2}$, the abundance of stacking faults results in high residual component of $\rho_{c}$ and good in-plane metallicity.

In summary, we have demonstrated that a delicate alternation of the interlayer crystalline structure of $2 \mathrm{H}-\mathrm{NbS}_{2}$, by introducing different polymorphs of the same atomic composition, dramatically affects the material's physical properties. In particular, the crystal's $2 \mathrm{H}$ stacking is occasionally disrupted by the 1T layers, which undergo a CDW instability. This then results in a triangular superlattice of David-star-shaped clusters, each hosting a lone spin at the centre. Such a texture of localised magnetic moments can be seen as a two-dimensional Kondo lattice, immersed into the metallic bulk of the $2 \mathrm{H}$ polytype. When the electric field or thermal gradient are then applied along the $c$ axis, electronic transport shows a pronounced Kondo effect manifesting as anomalies in the out-of-plane resistivity and Seebeck coefficient. But when they are applied within the plane, there is no sign of spin-dependent scattering. The observation of this highly anisotropic phenomenon occurring in a naturally formed heterostructure has been made possible, thanks to the careful tailoring of the crystal by FIB. Our work therefore shows the importance of adopting new experimental techniques in studying novel electronic materials, especially highly anisotropic Van der Waals structures.

\section{METHODS}

\section{Focused ion beam microfabrication}

Microstructured samples were extracted from monocrystalline flakes of TMDs. The starting crystals had the lateral size of the order of $1 \mathrm{~mm}$ and were at least $100 \mu \mathrm{m}$ thick. After identifying a clean region on a crystal's surface, free of cracks or buckling, a rectangular lamella was defined by milling away the surrounding material using an FEI Helios G4 Xe plasma FIB microscope. The typical dimensions of a lamella were around $120 \mu \mathrm{m} \times$ $60 \mu \mathrm{m} \times 5 \mu \mathrm{m}$ (with up to $20 \%$ variations in lengths between different samples), with the intermediate dimension corresponding to the extent along the $c$ axis of a crystal. The milling current for this stage was $60 \mathrm{nA}$, with $30 \mathrm{kV}$ column voltage. An FEI Helios G3 Ga FIB microscope was then used for polishing the surface of the lamella with a $1 \mathrm{nA}$ beam in order to ensure the parallelism of the two largest faces. After extraction, the lamella was glued to a sapphire substrate with a tiny amount of Araldite Rapid epoxy, keeping the external face exposed. Besides anchoring the lamella, the epoxy also formed a meniscus around it that smoothly connected the substrate's surface to the lamella's exposed face. The setup was then sputter coated with a $100 \mathrm{~nm}$ layer of gold. Next, the Ga FIB milling at $10 \mathrm{nA}$ was used for defining the probing electrodes by selectively removing the sputtered gold layer, and for patterning the lamella in order to form the current channel and voltage probing points. The procedure was concluded with polishing the exposed side faces of the sample with a $1 \mathrm{nA}$ ion beam in order to clean the surface of the re-deposited material and define the final dimensions of the device. Since the entire bottom face of the sample was rigidly attached to the substrate, differential thermal contraction and compressibility were expected to produce inhomogeneous stresses throughout the lamella. In our study, these stresses did not have a significant influence on the measured data. More detailed information about the FIB-assisted sample preparation can be found in the relevant review paper $^{7}$ and references therein.

\section{Resistivity measurements}

Resistivity was measured via the four-point technique with direct or alternating excitation currents in the $20-40 \mu \mathrm{A}$ range. Temperature sweeps' rate was limited to $1 \mathrm{~K} / \mathrm{min}$ for the ambient pressure and of $0.5 \mathrm{~K} / \mathrm{min}$ for the high-pressure measurements in order to reduce the thermal lag and gradients.

Resistivity at high pressure was measured using a piston cylinder cell produced by C\&T Factory. Daphne oil 7474 was used as a pressuretransmitting medium. Pressure was determined from the changes in resistance and superconducting transition temperature of a sample of $\mathrm{Pb}$ located next to the $2 \mathrm{H}-\mathrm{NbS}_{2}$ sample.

Measurements in high magnetic fields were conducted at the high magnetic field facilities in Grenoble (up to 34 T DC field) and Toulouse (up to $63 \mathrm{~T}$ pulsed field). Quantum Design PPMS was used for measurements in fields up to $14 \mathrm{~T}$.

\section{Seebeck coefficient measurements}

Seebeck coefficient was measured using an in-house setup. For the inplane Seebeck coefficient measurement, a thin and long sample was mounted on a ceramic bar. One end of the bar was connected to the thermal bath, while the other one had a resistive heater attached. A differential thermocouple was used to measure the temperature difference 
across the sample. The out-of-plane Seebeck coefficient measurement was performed using a setup displayed in Fig. $2 c$ and described in the corresponding caption.

\section{DATA AVAILABILITY}

The data that support the findings of this study are available from the authors (K.S., E.M., and L.F.) upon reasonable request. Data used for generating the plots can be found at https://doi.org/10.6084/m9.figshare.16720621.

Received: 20 July 2021; Accepted: 13 October 2021; Published online: 18 November 2021

\section{REFERENCES}

1. Novoselov, K. S. et al. Two-dimensional gas of massless Dirac fermions in graphene. Nature 438, 197-200 (2005).

2. Ellis, J. K., Lucero, M. J. \& Scuseria, G. E. The indirect to direct band gap transition in multilayered $\mathrm{MoS}_{2}$ as predicted by screened hybrid density functional theory. Appl. Phys. Lett. 99, 261908 (2011).

3. Wang, Z. et al. Evidence of high-temperature exciton condensation in twodimensional atomic double layers. Nature 574, 76-80 (2019).

4. Cao, Y. et al. Unconventional superconductivity in magic-angle graphene superlattices. Nature 556, 43-50 (2018).

5. Basov, D. N. \& Timusk, T. Electrodynamics of high-T superconductors. Rev. Mod. Phys. 77, 721-779 (2005).

6. Martino, E. et al. Preferential out-of-plane conduction and quasi-one-dimensional electronic states in layered $1 \mathrm{~T}-\mathrm{TaS}_{2}$. npj 2D Mater. Appl. 4, 7 (2020).

7. Moll, P. J. W. Focused ion beam microstructuring of quantum matter. Annu. Rev. Condens. Matter Phys. 9, 147-162 (2018).

8. Carmalt, C. J., Manning, T. D., Parkin, I. P., Peters, E. S. \& Hector, A. L. Formation of a new (1T) trigonal $\mathrm{NbS}_{2}$ polytype via atmospheric pressure chemical vapour deposition. J. Mater. Chem. 14, 290 (2004).

9. Shimakawa, M., Kawachi, K., Nishikawa, S. \& Hayashi, K. Structural stability of the 1T structure on transition-metal dichalcogenides. J. Solid State Chem. 129, 242-249 (1997).

10. Tresca, C. \& Calandra, M. Charge density wave and spin $1 / 2$ insulating state in single layer 1T-NbS, 2D Mater. 6, 035041 (2019).

11. Wang, W. et al. Charge density wave instability and pressure-induced superconductivity in bulk $1 \mathrm{~T}-\mathrm{NbS}_{2}$. Phys. Rev. B 102, 155115 (2020)

12. Guillamón, I. et al. Superconducting density of states and vortex cores of $2 \mathrm{H}$ $\mathrm{NbS}_{2}$. Phys. Rev. Lett. 101, 166407 (2008).

13. Yan, R. et al. Thickness dependence of superconductivity in ultrathin $\mathrm{NbS}_{2}$. Appl. Phys. Express 12, 023008 (2019).

14. Leroux, M., Rodière, P., Cario, L. \& Klein, T. Anisotropy and temperature dependence of the first critical field in $2 \mathrm{H}_{-}-\mathrm{NbS}_{2}$. Phys. B Condens. Matter 407, 1813-1815 (2012)

15. Leroux, M. et al. Anharmonic suppression of charge density waves in $2 \mathrm{H}_{-} \mathrm{NbS}_{2}$. Phys. Rev. B 86, 155125 (2012).

16. Lin, D. et al. Patterns and driving forces of dimensionality-dependent charge density waves in $2 \mathrm{H}$-type transition metal dichalcogenides. Nat. Commun. 11, 2406 (2020).

17. Heil, C. et al. Origin of superconductivity and latent charge density wave in $\mathrm{NbS}_{2}$. Phys. Rev. Lett. 119, 087003 (2017).

18. Jellinek, F., Brauer, G. \& Müller, H. Molybdenum and niobium sulphides. Nature 185, 376-377 (1960)

19. Fisher, W. G. \& Sienko, M. J. Stoichiometry, structure, and physical properties of niobium disulfide. Inorg. Chem. 19, 39-43 (1980).

20. Witteveen, $\mathrm{C}$. et al. Polytypism and superconductivity in the $\mathrm{NbS}_{2}$ system. Dalton Trans. 50, 3216-3223 (2021).

21. Katzke, $\mathrm{H}$. Stacking disorder in $2 \mathrm{H}-\mathrm{NbS}_{2}$ and its intercalation compounds $\mathrm{K}_{x}\left(\mathrm{H}_{2} \mathrm{O}\right)_{y} \mathrm{NbS}_{2}$. I. Description and model calculations of stacking faults in the host lattice $\mathrm{NbS}_{2}$. Z. Kristallogr. Cryst. Mater. 217, 127-130 (2002).

22. Leroux, M., Cario, L., Bosak, A. \& Rodière, P. Traces of charge density waves in $\mathrm{NbS}_{2}$. Phys. Rev. B 97, 195140 (2018).

23. Rossnagel, K. On the origin of charge-density waves in select layered transition-metal dichalcogenides. J. Phys. Condens. Matter 23, 213001 (2011).

24. Pfalzgraf, B. W. \& Spreckels, H. The anisotropy of the upper critical field $\mathrm{H}_{\mathrm{c} 2}$ and electrical resistivity in 2H-NbS . J. Phys. C. 20, 4359-4367 (1987).

25. Gunnarsson, O., Calandra, M. \& Han, J. E. Colloquium: saturation of electrical resistivity. Rev. Mod. Phys. 75, 1085-1099 (2003).

26. Naito, M. \& Tanaka, S. Electrical transport properties in $2 \mathrm{H}-\mathrm{NbS}_{2},-\mathrm{NbSe}_{2},-\mathrm{TaS}_{2}$ and $-\mathrm{TaSe}_{2}$. J. Phys. Soc. Jpn. 51, 219-227 (1982).
27. Goldsmid, H. J. Introduction to Thermoelectricity (Springer Science \& Business Media, 2009).

28. Aronov, B. L. \& Al'tshuler, A. G. Contribution to the theory of disordered metals in strongly doped semiconductors. Zh. Eksp. Teor. Fiz. 77, 2028-2044 (1979).

29. Lee, P. A. \& Ramakrishnan, T. V. Disordered electronic systems. Rev. Mod. Phys. 57, 287-337 (1985).

30. Xu, Y., Zhang, J., Cao, G., Jing, C. \& Cao, S. Low-temperature resistivity minimum and weak spin disorder of polycrystalline $\mathrm{La}_{2 / 3} \mathrm{Ca}_{1 / 3} \mathrm{MnO}_{3}$ in a magnetic field. Phys. Rev. B 73, 224410 (2006).

31. Altshuler, B. L., Khmel'nitzkii, D., Larkin, A. I. \& Lee, P. A. Magnetoresistance and Hall effect in a disordered two-dimensional electron gas. Phys. Rev. B 22, 5142-5153 (1980).

32. Forro, L., llakovac, V., Cooper, J. R., Ayache, C. \& Henry, J.-Y. Out-of-plane conductivity of $\mathrm{YBa}_{2} \mathrm{Cu}_{3} \mathrm{O}_{7-\delta}$. Phys. Rev. B 46, 6626-6629 (1992).

33. Chen, W., Andersen, B. M. \& Hirschfeld, P. J. Theory of resistivity upturns in metallic cuprates. Phys. Rev. B 80, 134518 (2009).

34. Alloul, H., Bobroff, J., Gabay, M. \& Hirschfeld, P. J. Defects in correlated metals and superconductors. Rev. Mod. Phys. 81, 45-108 (2009).

35. Kondo, J. Resistance minimum in dilute magnetic alloys. Prog. Theor. Phys. 32, 37-49 (1964)

36. Costi, T. A. et al. Kondo decoherence: finding the right spin model for iron impurities in gold and silver. Phys. Rev. Lett. 102, 056802 (2009).

37. Hanl, M. et al. Iron impurities in gold and silver: comparison of transport measurements to numerical renormalization group calculations exploiting nonAbelian symmetries. Phys. Rev. B 88, 075146 (2013).

38. Hewson, A. C. The Kondo Problem to Heavy Fermions (Cambridge University Press, 2003).

39. Zlatić, V., Costi, T. A., Hewson, A. C. \& Coles, B. R. Thermoelectric power of concentrated Kondo systems. Phys. Rev. B 48, 16152-16155 (1993).

40. Bickers, N. E., Cox, D. L. \& Wilkins, J. W. Self-consistent large-N expansion for normalstate properties of dilute magnetic alloys. Phys. Rev. B 36, 2036-2079 (1987).

41. Cooper, J. R., Vucic, Z. \& Babic, E. The thermoelectric power of AIMn alloys. J. Phys. F. Met. Phys. 4, 1489-1500 (1974).

42. Schilling, J. S. \& Holzapfel, W. B. Effect of pressure on the Kondo temperature of Cu:Fe - existence of a universal resistivity curve. Phys. Rev. B 8, 1216-1227 (1973).

43. Crone, J. \& Schilling, J. Effect of pressure on the Kondo temperatures of $\mathrm{Au}(\mathrm{Fe})$ and $\mathrm{Au}(\mathrm{Mn})$. Solid State Commun. 17, 791-794 (1975).

44. Goldhaber-Gordon, D. et al. From the Kondo regime to the mixed-valence regime in a single-electron transistor. Phys. Rev. Lett. 81, 5225-5228 (1998).

45. Nobukane, H., Tabata, Y., Kurosawa, T., Sakabe, D. \& Tanda, S. Coexistence of the Kondo effect and spin glass physics in Fe-doped $\mathrm{NbS}_{2}$. J. Phys. Condens. Matter 32, 165803 (2020)

46. Kačmarčík, J. et al. Specific heat measurements of a superconducting $\mathrm{NbS}_{2}$ single crystal in an external magnetic field: energy gap structure. Phys. Rev. B 82, 014518 (2010).

47. Pasquier, D. \& Yazyev, O. V. Charge density wave phase, Mottness, and ferromagnetism in monolayer $1 \mathrm{~T}-\mathrm{NbSe}_{2}$. Phys. Rev. B 98, 045114 (2018).

48. Calandra, M. Phonon-assisted magnetic Mott-insulating state in the charge density wave phase of single-layer 1T-NbSe 2 . Phys. Rev. Lett. 121, 026401 (2018).

49. Iqbal, Y., Hu, W.-J., Thomale, R., Poilblanc, D. \& Becca, F. Spin liquid nature in the Heisenberg J1-J2 triangular antiferromagnet. Phys. Rev. B 93, 144411 (2016).

50. Law, K. T. \& Lee, P. A. $1 \mathrm{~T}-\mathrm{TaS}_{2}$ as a quantum spin liquid. Proc. Natl Acad. Sci. USA 114, 6996-7000 (2017).

51. Lee, K. I. et al. Kondo effect in magnetic tunnel junctions. Phys. Rev. Lett. 98 107202 (2007)

52. Zheng, C., Shull, R. D., Chen, P. J. \& Pong, P. W. T. Kondo effect in magnetic tunnel junctions with an $\mathrm{AlO}_{x}$ tunnel barrier. Phys. Lett. A 380, 2237-2241 (2016).

53. Liu, M. et al. Monolayer $1 \mathrm{~T}-\mathrm{NbSe}_{2}$ as a correlated magnetic insulator. Preprint at https://arxiv.org/abs/2103.12948 (2021).

54. Vaňo, V. et al. Artificial heavy fermions in a van der Waals heterostructure. Preprint at https://arxiv.org/abs/2103.11989 (2021).

55. Ruan, W. et al. Evidence for quantum spin liquid behaviour in single-layer 1T$\mathrm{TaSe}_{2}$ from scanning tunnelling microscopy. Nat. Phys 17, 1154-1161 (2021).

56. Youbi, Z. E. et al. Fermiology and electron-phonon coupling in the $2 \mathrm{H}$ and $3 \mathrm{R}$ polytypes of $\mathrm{NbS}_{2}$. Phys. Rev. B 103, 155105 (2021).

57. Momma, K. \& Izumi, F. VESTA 3 for three-dimensional visualization of crystal, volumetric and morphology data. J. Appl. Crystallogr. 44, 1272-1276 (2011).

\section{ACKNOWLEDGEMENTS}

We would like to express gratitude to Dr. Osor S. Barišić (Institute of Physics in Zagreb), Prof. Andrew Mackenzie (MPI CPfS Dresden), Prof. Fakher Assaad (University of Würzburg), Prof. Frederic Mila (EPFL), Dr. Reza Zamani (EPFL) and particularly Prof. John Cooper (University of Cambridge) for valuable discussions and feedback. We 
acknowledge the support of Dr. Gaetan Giriat (EPFL) concerning the instrumentation and high-pressure cells, Dr. Maja Bachmann (MPI CPfS Dresden) for her assistance with FIB microfabrication, Dr. Wen Hua (David) Bi and Davor Tolj (EPFL) for their aid with the characterisation of crystals, Dr. Diego Pasquier (EPFL) for auxiliary numerical calculations. We acknowledge the support of the European Magnetic Field Laboratory (EMFL) for access to a 34 T static magnet at LNCMI-CNRS in Grenoble (Proposal: GMA04217), and access to a 70 T pulsed magnet at LNCMI-CNRS in Toulouse (Proposal: TSC05119). This study has been funded by the Swiss National Science Foundation through its SINERGIA network MPBH and grants No. 200021_175836 and PP00P2_170544. C. Putzke and P.J.W.M. acknowledge the support by the European Research Council (ERC) under the European Union's Horizon 2020 research and innovation programme (grant agreement No 715730) and the Max-Planck-Society. S.N.Z., Q.S.W. and O.V.Y. acknowledge support from NCCR Marvel.

\section{AUTHOR CONTRIBUTIONS}

E.M. and K.S. prepared and conducted resistivity and Seebeck coefficient measurements and wrote the manuscript together with L.F. C.P., M.K. and P.M. assisted with the FIB fabrication process. H.B. synthesised the crystals used in the study. D.L. assisted with resistivity measurements at the high DC magnetic field facility in Grenoble. M.L. and C.P. conducted resistivity measurements at the pulsed magnetic field facility in Toulouse. A.A. secured the magnet time for the experiments in Toulouse. H.K. and C.K. conducted the TEM study. S.Z., Q.W. and O.Y. provided theoretical support and the DFT data. L.F. is the project leader.

\section{COMPETING INTERESTS}

The authors declare no competing interests.

\section{ADDITIONAL INFORMATION}

Supplementary information The online version contains supplementary material available at https://doi.org/10.1038/s41699-021-00265-6.

Correspondence and requests for materials should be addressed to Edoardo Martino, László Forró or Konstantin Semeniuk.

Reprints and permission information is available at http://www.nature.com/ reprints

Publisher's note Springer Nature remains neutral with regard to jurisdictional claims in published maps and institutional affiliations.

(C) Open Access This article is licensed under a Creative Common Attribution 4.0 International License, which permits use, sharing, adaptation, distribution and reproduction in any medium or format, as long as you give appropriate credit to the original author(s) and the source, provide a link to the Creative Commons license, and indicate if changes were made. The images or other third party material in this article are included in the article's Creative Commons license, unless indicated otherwise in a credit line to the material. If material is not included in the article's Creative Commons license and your intended use is not permitted by statutory regulation or exceeds the permitted use, you will need to obtain permission directly from the copyright holder. To view a copy of this license, visit http://creativecommons. org/licenses/by/4.0/.

(c) The Author(s) 2021 